\title{
Alkaloid chemophenetics and transcriptomics of the Nicotiana genus
}

Kaminski K.P., Bovet L., Laparra H., Lang G., De Palo D., Sierro N., Goepfert S., Ivanov N.V.*

PMI R\&D, Philip Morris Products S.A., Quai Jeanrenaud 5, CH-2000 Neuchatel, Switzerland

*email: nikolai.ivanov@pmi.com

In order to discover detailed alkaloid biosynthesis pathways, it is important to first determine the quantity and composition of the alkaloid pool in Nicotiana species. It is also necessary to conduct research in which accurate targeted metabolite analysis is statistically correlated with gene expression analysis.

In this study, we determined for the first time the pyridine alkaloid content (nicotine, nornicotine, anabasine, anatabine, cotinine, and myosmine) of 58 species and 2 subspecies of the Nicotiana genus by ultra-high-performance liquid chromatography coupled with mass spectrometry. We observed a clear correlation between the Noctiflorae and Suaveolentes sections and their above-average accumulation of anabasine in the genus.

We analyzed the gene expression profiles of 60 Nicotiana species by mRNA sequencing for the first time. Hierarchical clustering of alkaloid biosynthesis pathway genes and alkaloid composition revealed patterns that clearly segregated the genus sections. The correlation of gene expression with alkaloid accumulation phenotypes was evident, such as the low putrescine methyltransferase expression in all species in the Noctiflorae and Suaveolentes sections and the clear correlation of nicotine demethylase with the conversion rates of nicotine to nornicotine in the majority of species.

Several additional correlations between alkaloid accumulation and gene expression values were identified, which makes this a fundamental study for future scientific exploration of the Nicotiana genus. 